\title{
Optical transmission matrix as a probe of the photonic strength
}

\author{
Duygu Akbulut, ${ }^{1, *}$ Tom Strudley, ${ }^{2, \dagger}$ Jacopo Bertolotti, ${ }^{1, \ddagger}$ Erik P. A. M. Bakkers, ${ }^{3,4}$ Ad Lagendijk, ${ }^{1}$ Otto L. Muskens, ${ }^{2}$ \\ Willem L. Vos, ${ }^{1}$ and Allard P. Mosk ${ }^{1, \S}$ \\ ${ }^{1}$ Complex Photonic Systems (COPS), MESA+ Institute for Nanotechnology, University of Twente, \\ P.O. Box 217, 7500 AE Enschede, The Netherlands \\ ${ }^{2}$ Faculty of Physical Sciences and Engineering, University of Southampton, Highfield, Southampton SO17 1BJ, United Kingdom \\ ${ }^{3}$ Department of Applied Physics, TU Eindhoven, Den Dolech 2, 5612 AZ Eindhoven, The Netherlands \\ ${ }^{4}$ Kavli Institute of Nanoscience, Delft University of Technology, 2600 GA Delft, The Netherlands
}

(Received 10 November 2015; published 12 October 2016)

\begin{abstract}
We demonstrate that optical transmission matrices (TMs) provide a powerful tool to extract the photonic strength of disordered complex media, independent of surface effects. We measure the TM of a strongly scattering $\mathrm{GaP}$ nanowire medium and compare the singular value density of the measured TM to a random-matrix-based wave transport model. By varying the transport mean free path and effective refractive index in the model, we retrieve the photonic strength. From separate numerical simulations we conclude that the photonic strength derived from TM statistics is insensitive to the surface reflection at rear surface of the sample.
\end{abstract}

DOI: 10.1103/PhysRevA.94.043817

\section{INTRODUCTION}

Scattering of waves in complex media is a phenomenon of basic scientific interest and of great importance for applications in mesoscopic electronics, imaging, photovoltaics, lighting, and optical communications [1-5]. In three-dimensional (3D) media, the photonic strength $S$ is a key parameter that describes the strength of scattering [6]. It quantifies how strongly the medium influences the propagation of waves. In disordered media $S$ quantifies how close a sample is to the Anderson localization transition [7,8]. In the diffusive regime photonic strength is given by $S=1 / k \ell$, where $\ell$ is the transport mean free path and $k$ the wave vector in the medium $[9,10] ; k=n_{\text {eff }} k_{0}$, with $n_{\text {eff }}$ the effective refractive index and $k_{0}$ the vacuum wave vector. At $k \ell \approx 1$, referred to as the Ioffe-Regel criterion [11], theory predicts a transition to three-dimensional localization [7,12], and indeed localization of ultrasound in three dimensions has been observed [13] and tantalizing indications of a localization transition of light in three dimensions have emerged [14,15]. In order to quantitatively study universal properties of scattering media in the diffusive regime and in the transition regime it is of critical importance to have a reliable probe of $S$ in the approach to the transition.

Existing methods to determine $S$ include the measuring of the enhanced backscattering cone [16,17] and of the total transmittance as a function of thickness [18]. A major limitation to these methods is that they are sensitive to the inevitable interfaces between the scattering medium and the surroundings with different refractive indices, giving rise to reflections. In

\footnotetext{
*Present address: ASML, Flight Forum 1900, 5657 EZ Eindhoven, The Netherlands.

${ }^{\dagger}$ Present address: Fianium Ltd., Ensign Way, Southampton, United Kingdom.

${ }^{\ddagger}$ Present address: Physics and Astronomy Department, University of Exeter, Stocker Road, Exeter EX4 4QL, United Kingdom.

${ }^{\S}$ Present address: Debye Institute for Nanomaterials Science, University of Utrecht, P.O. Box 80.000, 3508 TA Utrecht, The Netherlands; a.p.mosk@uu.nl
}

disordered media the interface layer often differs from the bulk, e.g., due to intrinsic sample growth inhomogeneities, exclusion effects, or processing steps. Hence, it can be difficult to estimate the reflections at the interfaces, which can show large sample to sample variability, compromising the determination of $S$.

Recently, it has been proposed that a scattering sample can be sensitively probed through the statistical properties of the transmission matrix (TM) [19,20]. The transmission matrix contains the amplitude transmission coefficients between a large number of incident and transmitted modes [19-23]. Intensive theoretical studies have been performed on the statistical properties of TMs of disordered waveguides, using random-matrix theories for wave transport [21], which are especially sensitive to the disorder inside the sample. An important tool in the analysis is the probability density of singular values, which are the square root of the transmission eigenvalues, of the TM. In works by Dorokhov and Mello et al. [24-26] this probability density was found to have a remarkable bimodal shape, containing a high density of exponentially small singular values (closed channels) as well as some singular values near unity, corresponding to open channels with almost perfect transmission $[27,28]$. Numerical work has confirmed and extended these theoretical results [29,30] and microwave and ultrasound TM measurements have confirmed the essential picture of the Dorokhov-Mello-Pereyra-Kumar (DMPK) theory [31-35].

In this paper we report using optical transmission matrix measurements to characterize the scattering medium itself, by probing the scattering strength in the bulk of the medium. The normalized singular value histogram of the transmission matrix of a sample (from here on referred to as the histogram) is shown to be a sensitive probe of the bulk scattering strength. Through numerical simulations we show that the histogram is insensitive to reflections at the exit interface of the sample, in contrast to other methods. We measure about $0.5 \%$ of the elements of the full transmission matrix of strongly scattering GaP nanowire mats and by inspecting the shape of the normalized singular value histogram, we retrieve the photonic strength $S$. 


\section{ANALYSIS AND NUMERICAL SIMULATION OF INTERFACE EFFECTS ON THE TRANSMISSION MATRIX}

Interface reflections can strongly modify transmission behavior and it cannot be assumed $a$ priori that these effects can be ignored or treated in the same manner as interface effects on the diffuse transmission. In this section we show through numerical simulations that the normalized singular value histogram is insensitive to surface reflections such as those caused by an air layer at the exit interface of the sample. Reflection at the interface of a scattering material, usually caused by index mismatch, reduces the angle-averaged transmission of the sample. In diffusion theory this is typically described as an increase in the diffuse extrapolation lengths [36,37]. The angle-and-ensemble-averaged transmission of the sample is written as

$$
\langle T\rangle=\frac{z_{i}+z_{e 1}}{L+z_{e 1}+z_{e 2}} .
$$

Here $L$ is the physical thickness of the sample and $z_{i}$ is the angle-averaged injection depth. This depth equals $z_{i}=$ $2 \ell / 3$ when averaged over angles between 0 and $\pi / 2$ and approaches $z_{i}=\ell$ in the case of close to normal incidence. The values $z_{e 1}$ and $z_{e 2}$ are the extrapolation lengths on the front and rear surfaces, respectively. In the absence of surface reflections, the extrapolation lengths $z_{e 1}$ and $z_{e 2}$ are approximately equal to $2 \ell / 3$. In Ref. [38], measurements on $\mathrm{GaP}$ nanowire transmission matrices were reported and the data were analyzed using a model that takes into account the increased extrapolation lengths due to surface reflections since a more elaborate analysis of the effect of surface reflections on the statistics of optical transmission matrices was missing at the time. In our sample, the largest reflection occurs at the exit surface, due to the presence of an air layer. We investigate the effect of interface reflections at this surface on the statistics of the TM. In some earlier studies, numerical and analytical results have been obtained on the effect of boundary reflections on transmission matrices $[30,39,40]$. In these studies it was shown that the distribution of transmission eigenvalues of the fully controlled TM is not very sensitive to surface reflection for moderate surface reflectivity. However, the effect of angle-dependent interface reflection on a filtered transmission matrix has not been investigated so far. Our study indicates that also for filtered TM, which is relevant for most experiments, surface reflections do not change TM statistics significantly. We performed finite-difference timedomain (FDTD) calculations, which fully take into account the details of reflection and filtering on the exit surface. We use the open-source MEEP package [41], taking about 2 CPU months to calculate a single TM on an i7-class processor. The computational setup is shown in Fig. 1.

The computational cell has size $W \times H \times Z$, where $W=$ $H=5 \mu \mathrm{m}$. The grid resolution is chosen as $30 \mu \mathrm{m}^{-1}$. In the center of the cell a zone of $W \times H \times L$ contains a disordered medium, which is generated by randomly placing small air spheres in a background medium with refractive index $n=3$.

We determine the mean free path of the numerical medium by fitting the energy density in an index-matched sample to diffusion theory. The energy density extrapolates to zero at the external extrapolation length of $0.71 \ell$ [2]. We find $\ell=0.6 \mu \mathrm{m}$

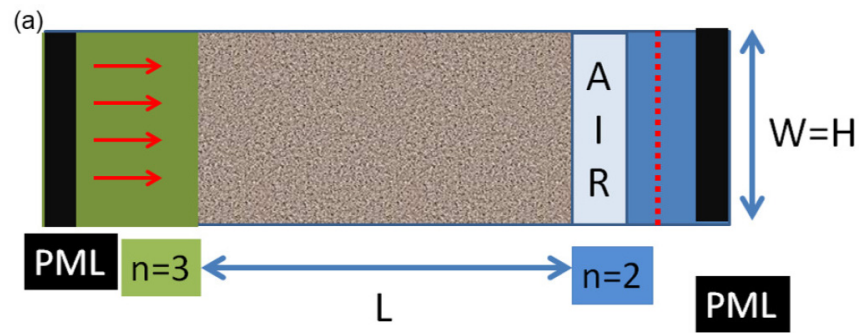

(b)

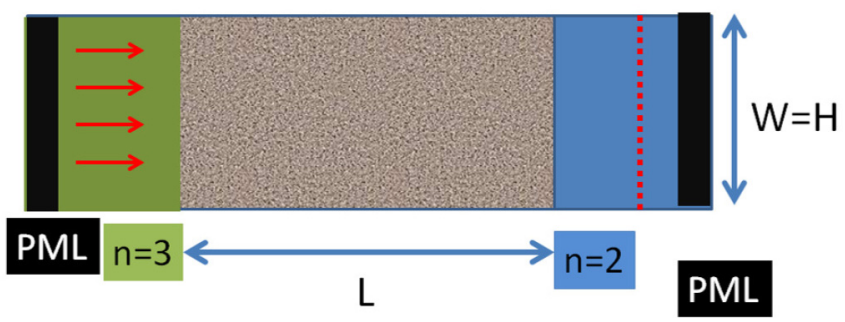

FIG. 1. (a) Schematic of a disordered medium with a layer of air on the exit side. (b) Schematic of a disordered medium with an index-matched layer on the exit side. Here PML denotes a perfectly matched layer. Red arrows show the location of the sources. The red dashed line is the detection plane.

at the wavelength used in the simulation, $\lambda=0.633 \mu \mathrm{m}$. The effective refractive index of the FDTD medium is found from effective-medium theory, $n_{\text {eff }}=1.8 \pm 0.2$, where the error bar arises from the differences between three different effectivemedium theories: Bruggeman's theory [42], Maxwell-Garnett theory [43], and Maxwell-Garnett theory for the inverse medium. From the mean free path and the effective index we calculate the photonic strength of the numerical medium as $S=0.09$.

The disordered medium is padded on both sides by index matching layers as follows. Perfectly matching layers (PMLs) that absorb any radiation are placed at the left and right of the cell. On the left of the disordered medium is a medium with refractive index $n=3$, modeling our bulk GaP substrate. On the right of the disordered medium is a medium of $n=2$, which is very close to the effective refractive index of the disordered medium, thus giving rise to very small internal reflection. Light is generated by a source on the left (modeling light incident through the substrate) and read out in a plane on the right just before the PML.

Two different computational configurations are considered in Fig. 1 to investigate the effect of the boundary conditions on the singular value histograms. In Fig. 1(a) a layer of air is present directly after the disordered medium, while in Fig. 1(b) the index matching medium with $n=2$ is in contact with the disordered region. The incident field is scanned in lateral dimensions over the interface at the left-hand side and the transmitted field is read out for each position and polarization configuration of the incident field. Each transmitted field is filtered in the reciprocal space to an effective numerical aperture (NA) of unity, as in the experiment.

The number of incident positions at which light is injected is 41 , for 2 orthogonal polarizations, and the effective number of modes of the transmitted field is about 400. Hence, the numerically generated transmission matrices have 82 singular 


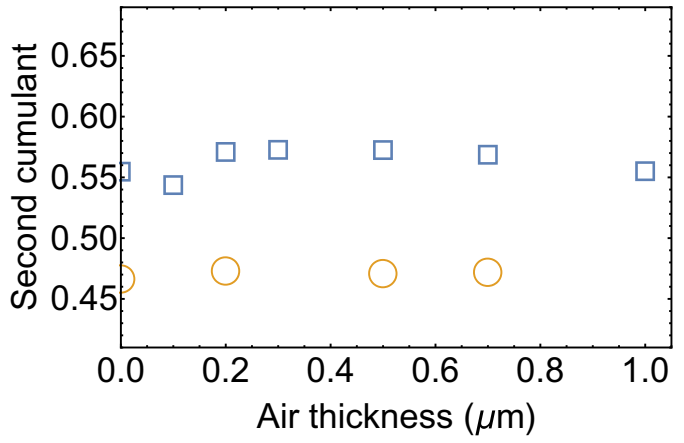

FIG. 2. Width of the normalized singular value histogram versus air layer thickness. Squares represent samples with thickness $L=5 \mu \mathrm{m}$ and circles samples with thickness $L=4 \mu \mathrm{m}$.

values, which is too low a value to reliably fit the histogram shape. In place of the histogram shape, we use the width of normalized singular value histogram, which increases with increased photonic strength, as a metric to study optical properties. A robust measure for the width of the normalized histogram is the second cumulant $C_{2}=\left\langle\widetilde{\lambda}^{2}\right\rangle-\langle\widetilde{\lambda}\rangle^{2}$, where $\{\widetilde{\lambda}\}$ are the normalized singular values so that $\left\langle\widetilde{\lambda}^{2}\right\rangle=1$.

The second cumulant of the singular value distribution is shown versus the air layer width in Fig. 2, for two different thicknesses of the scattering medium, $L=4$ and $5 \mu \mathrm{m}$. The samples with $L=5 \mu \mathrm{m}$ all show a value of $C_{2}$ of about 0.55 , irrespective of the air layer. The thinner samples with $L=4 \mu \mathrm{m}$ all have $C_{2} \approx 0.46$. This shows that $C_{2}$ is sensitive to the optical thickness of the scattering medium, but not significantly to the interface reflections caused by the air layer.

Next we quantitatively compare different methods of determining the photonic strength. For this purpose, we define the apparent photonic strength $S_{i}^{\text {app }}$ as the result of a measurement of the photonic strength obtained with a certain method $i$ that has not been corrected for the presence of the air layer. The quantity we compare is the correction factor $C=S^{\text {app }} / S$, with $S$ being the true photonic strength of the sample. For an ideal bulk-sensitive probe, $S^{\text {app }}=S$ and no correction for surface reflections needs to be done.

Correction factor for the backscatter cone. The width of the enhanced backscatter cone is widely used as a probe for the scattering strength, as for an index-matched sample the width is inversely proportional to the scattering strength [36]. For an index-mismatched sample with internal reflection the cone width is corrected by a factor

$$
C_{\mathrm{EBS}} \approx(1-\bar{R})
$$

where EBS denotes enhanced backscattering and $\bar{R}$ is the angle-averaged internal reflection coefficient [37].

Correction factor for total transmission measurement. When determining $S$ from diffuse transmission measurements, the apparent transport mean free path $\ell$ is retrieved by inverting Eq. (1). However, if internal reflections are present, the extrapolation lengths are increased by a factor close to $(1+\bar{R}) /(1-\bar{R})[36,37]$. Assuming an index mismatch only on the exit side, we find that the apparent photonic strength

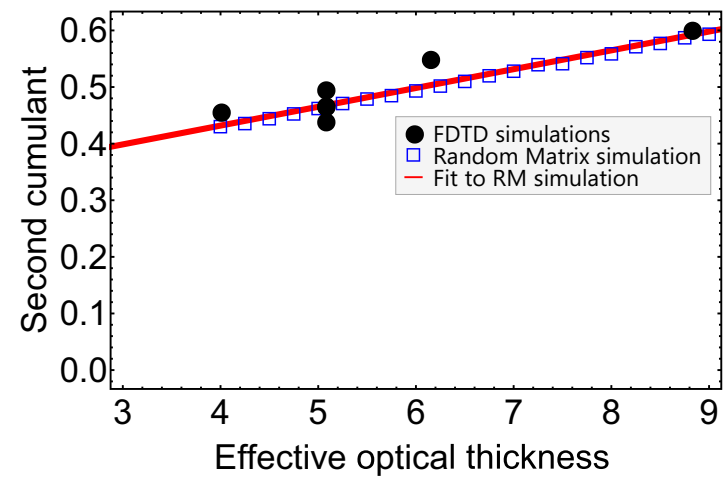

FIG. 3. Second cumulant of the normalized singular value histogram versus effective optical thickness $L_{\text {opt }}$ of an index-matched numerical sample. Closed circles show FDTD simulations, open squares show the random matrix calculation, and the solid line shows the linear fit to the random-matrix calculations.

from total transmission is off by a correction factor

$$
S_{\mathrm{TT}}^{\mathrm{app}} / S=1+\frac{4 \ell \bar{R}}{3 L(1-\bar{R})},
$$

where TT denotes total transmission.

Correction factor for TM statistics. To obtain the correction factor for TM statistics we use FDTD data. From Fig. 2 we see that the air layer has a very small effect on the second cumulant $C_{2}$ of the TM histograms. To quantify this small effect in terms of $S^{\text {app }}$ we obtain a heuristic relation between the TM histograms and the photonic strength of a sample in an index-matched environment.

In Fig. 3 we show FDTD calculations of the second cumulant $C_{2}$ versus the effective optical thickness $L_{\mathrm{opt}}=\langle T\rangle^{-1}$ of index-matched numerical samples. For these numerical samples, $\langle T\rangle=(4 \ell / 3) /(L+4 \ell / 3)$, with the injection depth and extrapolation lengths being equal to $2 \ell / 3$. Due to the small size of the samples, the FDTD data show some scatter.

For these index-matched numerical samples it is also possible to obtain the value of $C_{2}$ from random-matrix theory. This is achieved by numerically generating random matrices with DMPK statistics [2], with the transmission parameter $\langle T\rangle=1 / L_{\mathrm{opt}}$ as its input, and subsequently correcting these for overlap of the incident waves in the numerical sample and the sampling of only a part of the transmission matrix, in a procedure exactly analogous to our data analysis model, as described in Sec. IV. The resulting data are shown alongside the FDTD data in Fig. 3. It can be seen that the FDTD data and the random-matrix model agree very well.

The results of the random-matrix model are heuristically fit with a linear function

$$
C_{2}=\alpha+\beta L_{\mathrm{opt}} .
$$

Here $\alpha$ is an offset value and $\beta$ is the sensitivity of the $C_{2}$ to the optical thickness. We note that the heuristic values of $\alpha$ and $\beta$ depend strongly on the fraction of incident and transmitted modes controlled and measured and on the overlap of incident waves. From the fit in Fig. 3 we obtain $\alpha=0.30 \pm 0.01$ and $\beta=0.033 \pm 0.001$. Inverting the heuristic relation and using the expression $1 / L_{\mathrm{opt}}=(4 \ell / 3) /(L+4 \ell / 3)$, we find the 


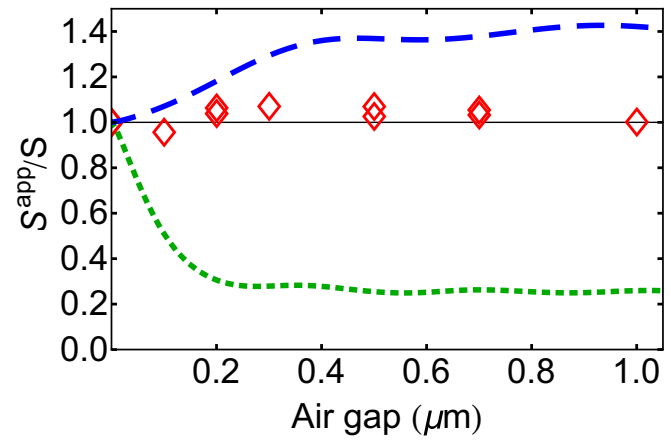

FIG. 4. Calculated apparent photonic strength for three methods normalized by the true value of $S$ for a sample with $L=10 \ell$ and effective refractive index $n_{\text {eff }}=1.8$. An air layer $(n=1)$ is present between the exit surface of the sample and the index matching medium. Red diamonds show $S_{\mathrm{TM}}^{\mathrm{app}}$ for TM statistics, as calculated by 3D FDTD; the blue dashed line shows $S_{\mathrm{TT}}^{\text {app }}$, as calculated for the total transmission method; and the green dotted line shows $S_{\mathrm{EBS}}^{\mathrm{app}}$, as calculated for the EBS cone width method.

apparent photonic strength as a function of $C_{2}$,

$$
S^{\mathrm{app}}=\frac{4}{3 \beta L n_{\mathrm{eff}} k_{0}}\left(C_{2}-\alpha-\beta\right) .
$$

Using Eq. (5) on an index-matched calculation with the same parameters yields the true photonic strength $S$. The ratio $S^{\text {app }} / S$ is given by

$$
S_{\mathrm{TM}}^{\mathrm{app}}\left(d_{\mathrm{air}}\right) / S=\frac{C_{2}\left(d_{\mathrm{air}}\right)-\alpha-\beta}{C_{2}(d=0)-\alpha-\beta} .
$$

Here TM denotes TM statistics and $C_{2}(d=0)$ signifies the index-matched calculation with an air layer thickness $d=0$.

In Fig. 4 we show the apparent photonic strength for the TM statistics $S_{\mathrm{TM}}^{\mathrm{app}}$, as obtained from 3D FDTD simulations, in addition to the corresponding value calculated for the backscattering cone and total transmission methods for the same sample geometry. We see that $S_{\mathrm{TM}}^{\mathrm{app}}$ is close to $S$ for any air layer thickness. In contrast, the apparent photonic strengths that result from total transmission $S_{\mathrm{TT}}^{\mathrm{app}}$ and from the enhanced backscattering cone width $S_{\mathrm{EBS}}^{\mathrm{app}}$ strongly deviate from the true $S$ for air layers thicker than $100 \mathrm{~nm}$. This deviation indicates that when one uses total transmission or enhanced backscattering cone data to probe the photonic strength, a major correction is required if an air layer or other reflecting surface is present. Since the apparent photonic strength for those methods is a steep function of the air layer thickness, precise knowledge of the exact surface condition is essential to correct for errors in the range of $50 \%$ or even beyond. In contrast, in the case of TM statistics no correction is needed since the apparent $S$ is very close to the true value. This leads to the remarkable result that the histogram of a sample with an air layer is essentially equal to that of the index matched sample, even if the average diffuse transmission is very different.

\section{EXPERIMENT}

The samples that we studied in this paper are disordered semiconductor nanowire mats, which are extremely strongly scattering samples $[10,44]$. Nanowires were grown using metal-organic vapor epitaxy on a GaP (100) substrate, with a refractive index of 3.32 at $\lambda=632.8 \mathrm{~nm}$ wavelength [45] and reach to a length of up to $6.4 \mu \mathrm{m}$ [10]. To obtain a maximally disordered arrangement, the nanowires were crushed by applying pressure with a glass slide. The average diameter of the crushed nanowires is $300 \mathrm{~nm}$ with a standard deviation of $50 \mathrm{~nm}$. We estimate the thickness of our sample to be $6 \mu \mathrm{m}$. In samples similar to the ones studied here, a transport mean free path as low as $\ell=0.3 \mu \mathrm{m}$ at $\lambda=632.8 \mathrm{~nm}$ was observed [44]. The effective refractive index of the nanowire mat is $n_{\text {eff }}=1.9 \pm 0.4$, estimated using Bruggeman's formula [42], where the error margin arises from the uncertainty in the volume fraction, $\phi=0.44 \pm 0.15$ as estimated from scanning electron microscopy images. The glass slide was left pressed onto the nanowire mat to allow imaging with an oil immersion objective during the transmission matrix measurements. However, in some samples a sub- $\mu \mathrm{m}$ air layer of inhomogeneous thickness developed between the nanowires and the glass. When using TM statistics, even the strong internal reflections caused by such an air layer do not impede accurate measurements of the bulk scattering strength.

Our experimental setup is shown in Fig. 5. A spatial light modulator (SLM) (Holoeye Pluto) is used to scan the focused spot of a laser (wavelength $632.8 \mathrm{~nm}$, power $5 \mathrm{~mW}$ ) over the surface of the nanowire layer. For each position of the focused spot the transmitted light field is imaged using off-axis holography $[46,47]$. The sample is oriented with the $\mathrm{GaP}$ substrate on the side of the incident light. In order to reduce the aberrations caused by focusing through the $300-\mu \mathrm{m}$-thick GaP slab, we limit the NA of the illumination objective to 0.6. In addition, we use its cover glass correction ring to compensate for the aberration that the thick slab induces. The light transmitted through the nanowire layer is collected with an oil immersion objective with an NA of 1.42. By use of high-NA microscope objectives and by combining measurements of two polarization channels on the incident side, we address as much as $5 \%$ of the incident modes and capture $10 \%$ of the transmitted modes on the $\left(12.8 \times 12.8 \mu \mathrm{m}^{2}\right)$ effective area $A$ of the sample, where the effective area is defined as having a width equal to the average of the widths of probed area on incident surface and the full width at half maximum

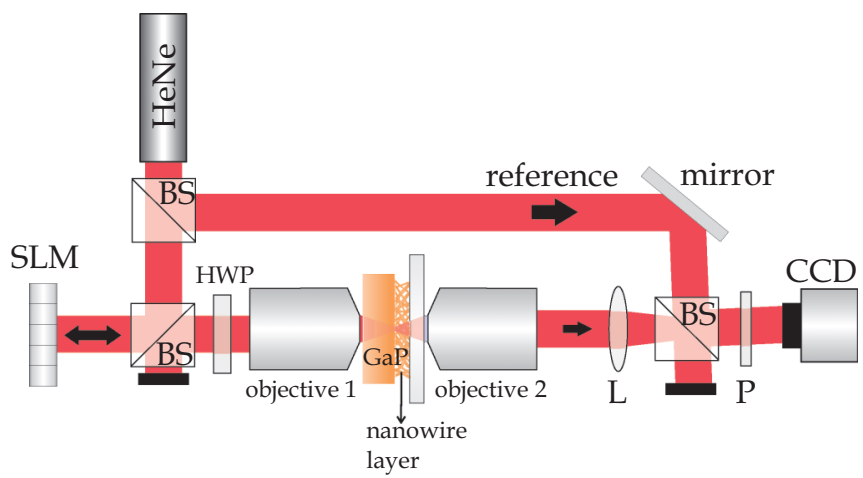

FIG. 5. Experimental setup: HeNe, laser; BS, 50:50 beam splitter; SLM, phase-only spatial light modulator; HWP, half-wave plate; GaP, sample substrate; nanowire layer, sample; objective 1, 40× 0.6-NA objective; objective 2, 60× 1.42-NA oil immersion objective; L, 500mm-focal-length lens; $\mathrm{P}$, polarizer; $\mathrm{CCD}$, camera sensor. 
(a)

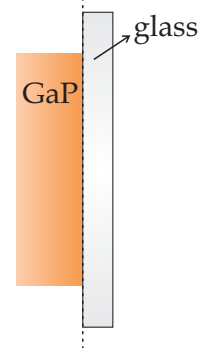

(b)

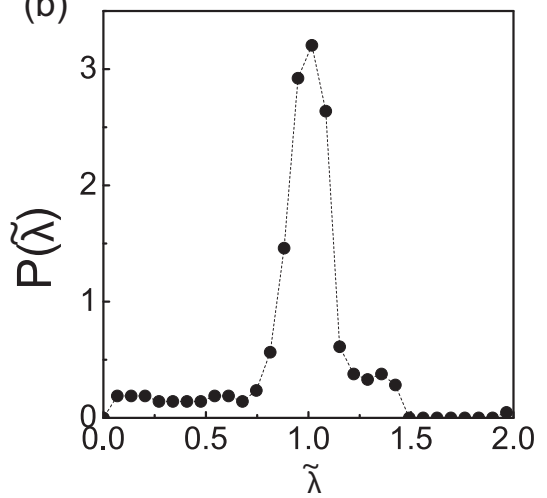

FIG. 6. (a) Schematic cross section through the reference sample consisting of a blank GaP slab glued to a glass slide. (b) Transmission singular value histogram of $T_{0}$, the TM of the reference GaP substrate without nanowires, normalized so that $\sqrt{\left\langle\widetilde{\lambda}^{2}\right\rangle}=1$. The histogram levels are connected by lines to guide the eye.

(FWHM) of the diffuse transmitted spot. While it is desirable to measure a large part of the TM, it is essential to not introduce unwanted overlap of incident waves, which leads to spurious correlations in the TM and affects the shape of the singular value histogram. To measure a sufficiently large part of the TM so that correlations in the TM can manifest themselves in the singular value histogram, without significant overlap of incident fields, we scanned the incident spot in a checkerboard pattern for each incident polarization. The spacing between nearest-neighbor spots was $673 \pm 25 \mathrm{~nm}$, which is about one wavelength.

The response of the optical setup is measured by repeating the whole measurement procedure with a nonscattering blank sample, which is a GaP slab glued to a glass slide, as shown in Fig. 6(a). The incident fields are focused at the interface between the GaP slab and the glass slide, as shown by the dashed line, and are imaged onto the CCD camera from the same plane. In Fig. 6(b) we show the singular value histogram of $T_{0}$, which is the matrix recorded using the calibration sample shown in Fig. 6(a). The singular values are normalized so that their second moment is equal to 1 . The singular value histogram shows a peak centered at the singular value of 1.02 with a full width at half maximum of 0.23 . A small but significant density of low singular values is observed. This is attributed to overlap between the fields transmitted through the reference sample. Two isolated singular values are observed at 1.96 and 2.91 (not shown). The two isolated high singular values are present in all sets of recorded matrices and are attributed to fields that are present as an offset in all recorded fields such as a small reflection from the front window of the SLM. These two outlying singular values have negligible effect on the normalization and are excluded from further data analysis. The finite width of the histogram is attributed to an interplay of detection noise, fluctuations in the spot intensity, and overlap between incident spots.

In transmission matrix measurements on samples in slab geometry, the illuminated area on the rear surface of the sample is larger than that on the front surface due to diffusion of light. In contrast, the DMPK model is based upon a straight

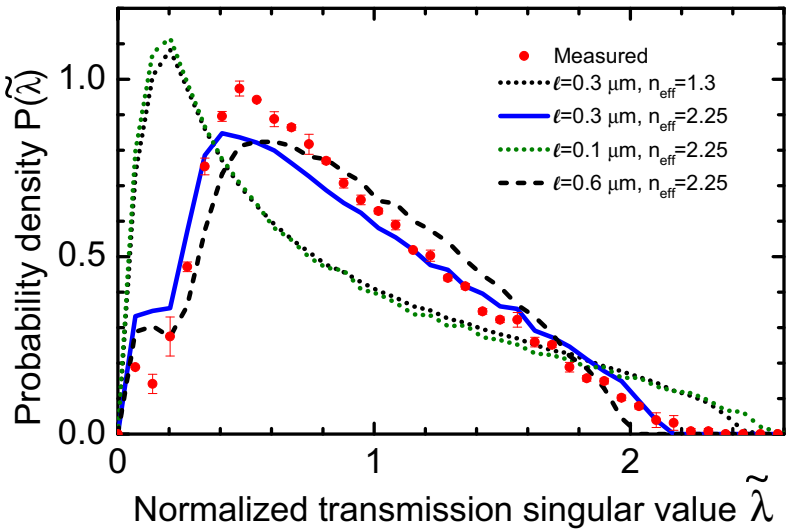

FIG. 7. Normalized singular value histograms obtained from the experiment (red circles, mean value of 3 experiments; error bars, standard error of the mean) and model with a priori estimated parameters $\ell=0.3 \mu \mathrm{m}$ and $n_{\text {eff }}=1.3$ (black dotted curve), $\ell=$ $0.3 \mu \mathrm{m}$ and $n_{\text {eff }}=2.25$ (blue solid curve), $\ell=0.1 \mu \mathrm{m}$ and $n_{\text {eff }}=$ 2.25 (green dotted curve), and $\ell=0.6 \mu \mathrm{m}$ and $n_{\text {eff }}=2.25$ (black dashed curve). All model histograms are a mean of 20 different histograms generated with independent random matrices.

waveguide model with a constant well-defined cross section. To make a mapping between our experimental situation and the idealized theoretical one we first define the widths of the illuminated areas on the input and output surfaces. The width of the input illumination is known, as the input patterns consist of spots on a staggered grid. The spatial width of the output pattern is found from diffusion theory. However, in the data analysis a slightly larger field of view is sampled to take into account spreading of light, e.g., due to the presence of an air layer at the surface. Ideally, selecting a field of view that is much larger than the illuminated area does not change the statistics of the sampled light. However, in an experiment when a very large field of view is selected, the accumulated detection noise starts to distort the histogram. In order to avoid accumulating a large amount of detection noise, we choose a field of view of $13.9 \mu \mathrm{m}$ width, which is equal to the FWHM of the total intensity pattern of all detected fields.

For the samples used in this study, it was discovered that the nanowires and the glass slide are not always in contact, while some nanowires are broken and stuck on the glass slide. We think this plays a role in the inhomogeneous distribution of light observed in momentum space. In order to have a welldefined field distribution in momentum space independent of the presence of an air gap between the nanowires and the glass slide, we apply a digital aperture with an effective NA of 1 to each measured field [38].

In Fig. 7 we show the measured histogram of a disordered $\mathrm{GaP}$ nanowire mat. The singular values $\{\widetilde{\lambda}\}$ are normalized so that $\left\langle\widetilde{\lambda}^{2}\right\rangle=1$, i.e., the mean square of the singular values is normalized to unity. The histogram has a peak at $\widetilde{\lambda}=$ $0.49_{-0.05}^{+0.06}$ and a slightly concave tail that extends up to $\widetilde{\lambda}=2.3$, corresponding to an estimated absolute intensity transmission of 0.4. We quantitatively analyze the experimentally obtained histogram in Fig. 7 by comparing it to histograms generated numerically using the DMPK theory. 


\section{NUMERICAL EVALUATION OF THE DMPK MODEL}

In order to make a meaningful comparison, we apply the waveguide-based DMPK theory to our slab-type samples and take into account experimental effects such as the transmission through the optics and the substrate. Even more importantly, in any experiment the TM is filtered, i.e., only a finite field of view and only part of the solid angle can be sampled, restricting the number of input and output modes accessible to the experimenter. This filtering strongly affects the shape of the singular value probability density $[48,49]$. It was shown in Ref. [49] that when the fraction of probed modes is even slightly smaller than 1 , the peak corresponding to the open channels is lost. When the fraction is reduced much further, the characteristic probability density of an uncorrelated random matrix is obtained [50], as is the case in Ref. [19]. In later experiments with a very large number of probed modes, the histogram shape was found indeed to deviate from that of an uncorrelated random matrix [23]. In our study, we probe a sufficient number of modes to observe deviations in the histogram shape from that of the uncorrelated random matrix, which we utilize to inspect the photonic strength of the sample under study. Importantly, we use reference measurements to take into account any spurious correlations that are caused by overlapping incident waves. Thereby we quantitatively retrieve the photonic strength from the TM.

We numerically evaluate the DMPK theory as follows. We first generate two uncorrelated unitary matrices $U_{1}$ and $U_{2}$, sampled Haar uniformly from $U(N)$ following a standard procedure described, e.g., in [51]. We then generate a diagonal matrix $D$ with elements sampled from the DMPK distribution and calculate $U_{1} D U_{2}$. The internal TM of the sample is a large matrix of size $8000 \times 8000$ with a DMPK singular value density and an average internal transmission of $\langle T\rangle=$ $\left(z_{i}+z_{e}\right) /\left(L+2 z_{e}\right)$, where $z_{i} \approx \ell$ is the effective injection depth and $z_{e} \approx 2 \ell / 3$ is the extrapolation length [52,53]. We emphasize that to calculate the histogram we must here use the extrapolation length for an index-matched sample as according to Sec. II this leads to the correct histogram even in the case where an index-mismatching air layer is present. Next we take into account the filtering of the TM in an experiment. The total number of transversal modes to take into account $N_{\mathrm{wg}}$ follows from approximating the sample as a waveguide with a cross-section area $A$, with the width taken as the average of the width of the probed area on the incident surface and the FWHM width the diffuse transmitted spot. This results in $N_{\text {wg }}=\left(2 \pi A n_{\text {eff }}^{2}\right) / \lambda^{2}$, with $\lambda$ the free space wavelength [54]. On the input side, the filtering ratio is the ratio of the number of probed modes to $N_{\mathrm{wg}}$. On the output side the filtering is due to the detection NA, therefore the filtering ratio is $\mathrm{NA}^{2} / 2 n_{\text {eff }}^{2}$. The factor 2 in the denominator is due to detection being made for a single polarization. In our model, the filtering is done by cropping the internal TM by the appropriate ratios. As the filtering is asymmetric, the TM is rectangular. Finally, we take into account the propagation through the optics and substrate by multiplying the model TM by a matrix $\widetilde{T}_{0}$ that has the same singular value distribution as the measured reference transmission matrix $T_{0}$, but is rescaled to have dimensions $N_{\text {in }} \times N_{\text {in }}$. The effects of the overlap between different incident fields and the multiplicative noise are included in the model via multiplication by $\widetilde{T}_{0}$.
We note that since the imperfections in the optical system do not lead to reflections, we can directly multiply transmission matrices instead of using the more complicated composition rule for $S$ matrices that is appropriate when multiple reflections cannot be ignored [55]. Additive detection noise is found to be small compared to the multiplicative noise components. Its inclusion in the model does not yield significantly different results. The described approach allows us to generate singular value histograms that include the basic physical effects as well as the characteristics of our measurement apparatus. The results of the model can be compared directly to the experimental results.

The histogram obtained from the model for a realistic estimate of the sample parameters based on previous data [44], $n_{\text {eff }}=2.25$ and $\ell=0.3 \mu \mathrm{m}$, is shown in Fig. 7, along with the histograms obtained for an unrealistically high $\ell=0.6 \mu \mathrm{m}$ and for an unrealistically low $\ell=0.1 \mu \mathrm{m}$, while retaining the same $n_{\text {eff }}$. In addition, the histogram for $n_{\text {eff }}=1.3$ and $\ell=0.3 \mu \mathrm{m}$ is given to show the histogram for an unrealistically low $n_{\text {eff }}$. The model and the experimental histograms are in good agreement for $\ell=0.3 \mu \mathrm{m}$ and $n_{\text {eff }}=2.25$. Both curves are asymmetric in shape with a sharp rise at low singular values to reach a peak near $\tilde{\lambda}=0.4$. After the peak, both histograms decrease in a slightly concave manner, with the experimental histogram having a higher slope, both reaching 0 counts near $\widetilde{\lambda}=2.3$. The model histogram with the longer mean free path shows an obviously more convex shape than the experimental data and the model histogram with the shorter mean free path is more pronouncedly concave, indicating that the photonic strength can be retrieved from the shape of the histogram.

\section{ESTIMATION OF THE PHOTONIC STRENGTH}

To find the parameters that offer the best match between our model and experiments we evaluate the model for a range of the only adjustable parameters, the mean free path $\ell$ and the effective index $n_{\text {eff }}$. In Fig. 8 we show the distance metric $\chi^{2}$ between model and experimental data for a rectangular domain encompassing the likely range of $\ell$ and $n_{\text {eff }}$. Here $\chi^{2}$ is defined as

$$
\chi^{2}=\sum_{k=k^{\prime}}^{M} \frac{\left|\mathcal{H}_{1}(k)-\mathcal{H}_{2}(k)\right|^{2}}{\mathcal{H}_{1}(k)+\mathcal{H}_{2}(k)+\varepsilon},
$$

with $k$ the histogram bin index, $k^{\prime}$ the histogram bin index of the bin with maximum counts, and $M$ the total number of histogram bins [56]. In addition, $\mathcal{H}_{1}(k)$ is the number of counts in the $k$ th numerical histogram bin; $\mathcal{H}_{2}(k)$ is the number of counts in $k$ th experimental histogram bin. The offset $\varepsilon=10^{-32}$ is included to avoid division by zero. The low singular values that lie to the left of the peak in the histogram are not taken into account as this part of the histogram is found to be sensitive to detection noise [38].

The region of minimum $\chi^{2}$ is a diagonal valley running from high $\ell$ and low $n_{\text {eff }}$ to low $\ell$ and high $n_{\text {eff }}$. Remarkably, this valley approximately tracks the curves of constant $S$. While we did not a priori expect this shape of the valley, this is a fortunate situation as it allows us to accurately determine $S$. While the fitting of model histograms to the experimental histograms gives little independent information 


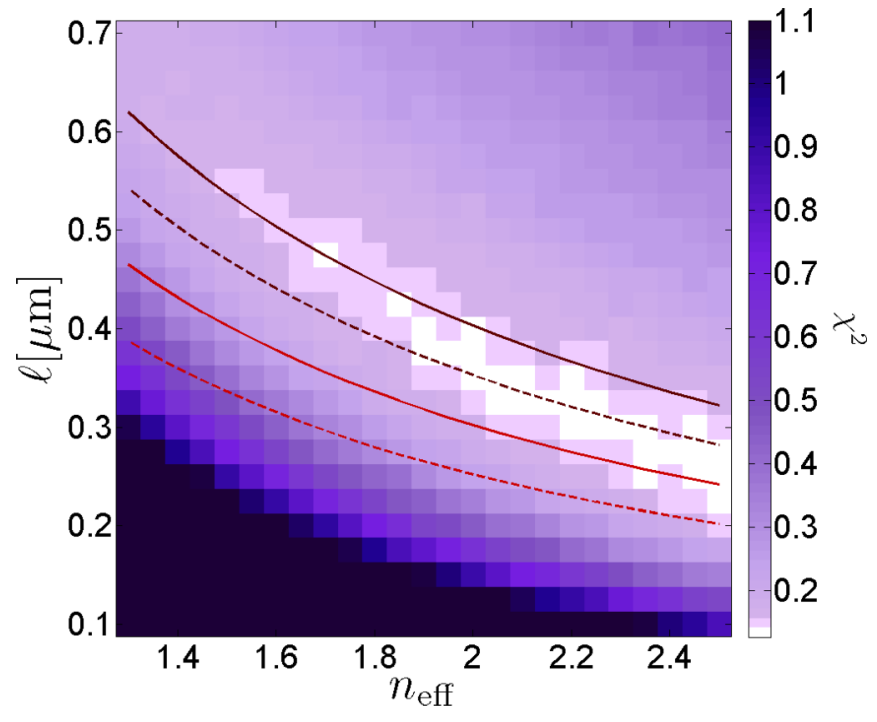

FIG. 8. Map of the distance metric $\chi^{2}$ between the numerical model and the experimental data, as a function of the parameters $\ell$ and $n_{\text {eff }}$. The color scale saturates at $\chi^{2}=1.1$. The white region corresponds to the best agreement between experiment and the model. The dashed light red curve shows $k \ell=5$, the solid light red curve $k \ell=6$, the dashed dark red curve $k \ell=7$, and the solid dark red curve $k \ell=8$.

on $\ell$ and $n_{\text {eff }}$, we find that the photonic strength can be accurately determined to be $S=0.14$, or equivalently $k \ell=7$, with a $20 \%$ error estimate. The error margin is determined by considering the minimum and maximum of $S$ in the region where $\chi^{2}-\chi_{\min }^{2}<3 \sigma$, where $\chi_{\min }^{2}$ is the global minimum and $\sigma$ is the standard deviation of $\chi_{\min }^{2}$ as obtained from the comparison between the average experimental histogram and each model histogram. This procedure estimates the statistical error due to the parameter estimation procedure and the slight deviation of the valley of best fit from the constant $S$ curves. Furthermore, the uncertainty in the thickness of the sample, which is on the order of $8 \%$, is included in the $20 \%$ error estimate. The value of $S$ obtained here is compatible with the measurements reported in Ref. [44] and the $n_{\text {eff }}$ values estimated from the filling fraction. The level of uncertainty reached here is good compared to other methods of measuring the photonic strength, such as enhanced backscattering or total transmission measurements, considering that no $a$ priori assumption about $n_{\text {eff }}$ is made and that the method is not sensitive to surface effects.
Our apparatus was not calibrated to measure absolute intensity transmission. However, using diffusion theory and the best estimates for the effective index and mean free path, the average intensity transmission $\langle T\rangle$ can be estimated to be 0.08 , consistent with the values previously reported for similar samples in Ref. [44]. As a result, the highest measured normalized channel transmission $\widetilde{\lambda}=2.3$ corresponds to an estimated absolute intensity transmission of 0.4 .

\section{CONCLUSION}

In summary, we have demonstrated that the measurement of the transmission matrix is a powerful method to characterize the properties of a scattering material. In particular, we have shown that the transmission matrix measurements can be modeled with random-matrix wave transport theory to reliably yield the photonic strength as the only relevant free parameter. Through separate FDTD calculations we have shown that this approach is surprisingly robust to internal reflections by surface layers. The method is therefore very well suited to investigate mesoscopic samples with rough surfaces such as photonic glasses [57], powders, and disordered photonic band gap crystals, as well as 3D ultrasound media [13]. The precise characterization of the bulk scattering strength that our method provides is a prerequisite to a quantitative understanding of the approach to the Anderson transition in such media. An analytic description of the influence of angular distribution by boundary reflections, supplementing the expressions for filtered transmission matrices [49], would likely increase the range of conditions in which our method can be applied. With a higher signal to noise ratio, or when capturing an even larger part of the matrix, transmission matrix measurements may allow us to precisely detect deviations from diffusion in the critical regime.

\section{ACKNOWLEDGMENTS}

We thank Pepijn Pinkse, Klaus Boller, Arthur Goetschy, and Douglas Stone for insightful discussions and Cornelis Harteveld for technical assistance. This work is part of the research program of the Stichting voor Fundamenteel Onderzoek der Materie, which is financially supported by the Nederlandse Organisatie voor Wetenschappelijk Onderzoek. We acknowledge support from ERC Grant No. 279248, NWOVici, STW, the Royal Society, and EPSRC through fellowship EP/J016918/1.
[1] A. Ishimaru, Wave Propagation and Scattering in Random Media (Academic, New York, 1978).

[2] E. Akkermans and G. Montambaux, Mesoscopic Physics of Electrons and Photons (Cambridge University Press, Cambridge, 2007).

[3] Tutorials in Complex Photonic Media, edited by M. McCall, M. Noginov, N. Zheludev, and G. Dewar (SPIE, Bellingham, 2009).

[4] D. S. Wiersma, Nat. Photon. 7, 188 (2013).
[5] M. Ghulinyan and L. Pavesi (eds.), Light Localisation and Lasing: Random and Quasi-Random Photonic Structures (Cambridge University Press, Cambridge, UK, 2015).

[6] W. L. Vos, A. Lagendijk, and A. P. Mosk, in Ref. [5], Chap. 1, p. 1; also available from arXiv:1504.06808.

[7] P. W. Anderson, Phys. Rev. 109, 1492 (1958).

[8] A. Lagendijk, B. van Tiggelen, and D. S. Wiersma, Phys. Today 62(8), 24 (2009).

[9] O. L. Muskens and A. Lagendijk, Opt. Express 16, 1222 (2008). 
[10] O. L. Muskens, S. L. Diedenhofen, B. C. Kaas, R. E. Algra, E. P. A. M. Bakkers, J. Gómez Rivas, and A. Lagendijk, Nano Lett. 9, 930 (2009).

[11] A. F. Ioffe and A. R. Regel, Prog. Semicond. (London) 4, 237 (1960).

[12] P. W. Anderson, Philos. Mag. B 52, 505 (1985).

[13] H. Hu, A. Strybulevych, J. Page, S. Skipetrov, and B. Van Tiggelen, Nat. Phys. 4, 945 (2008).

[14] D. S. Wiersma, P. Bartolini, A. Lagendijk, and R. Righini, Nature (London) 390, 671 (1997).

[15] M. Störzer, P. Gross, C. M. Aegerter, and G. Maret, Phys. Rev. Lett. 96, 063904 (2006).

[16] M. P. Van Albada and A. Lagendijk, Phys. Rev. Lett. 55, 2692 (1985).

[17] P. E. Wolf and G. Maret, Phys. Rev. Lett. 55, 2696 (1985).

[18] N. Garcia, A. Z. Genack, and A. A. Lisyansky, Phys. Rev. B 46, 14475 (1992).

[19] S. M. Popoff, G. Lerosey, R. Carminati, M. Fink, A. C. Boccara, and S. Gigan, Phys. Rev. Lett. 104, 100601 (2010).

[20] E. G. van Putten and A. P. Mosk, Physics 3, 22 (2010); A. Dogariu, ibid. 3, 67 (2010); E. G. van Putten and A. P. Mosk, ibid. 3, 68 (2010).

[21] C. W. J. Beenakker, Rev. Mod. Phys. 69, 731 (1997).

[22] M. Kim, Y. Choi, C. Yoon, W. Choi, J. Kim, Q.-H. Park, and W. Choi, Nat. Photon. 6, 581 (2012).

[23] H. Yu, T. R. Hillman, W. Choi, J. O. Lee, M. S. Feld, R. R. Dasari, and Y. K. Park, Phys. Rev. Lett. 111, 153902 (2013).

[24] O. N. Dorokhov, Solid State Commun. 51, 381 (1984).

[25] P. A. Mello, P. Pereyra, and N. Kumar, Ann. Phys. (NY) 181, 290 (1988).

[26] Y. Imry, Europhys. Lett. 1, 249 (1986).

[27] J. B. Pendry, A. MacKinnon, and A. B. Pretre, Physica A 168, 400 (1990).

[28] J. B. Pendry, Physics 1, 20 (2008).

[29] K. A. Muttalib, J. L. Pichard, and A. D. Stone, Phys. Rev. Lett. 59, 2475 (1987).

[30] Y. V. Nazarov, Phys. Rev. Lett. 73, 134 (1994).

[31] Z. Shi and A. Z. Genack, Phys. Rev. Lett. 108, 043901 (2012).

[32] M. Davy, Z. Shi, J. Wang, and A. Z. Genack, Opt. Express 21, 10367 (2013).

[33] Z. Shi, J. Wang, and A. Z. Genack, Proc. Natl. Acad. Sci. USA 111, 2926 (2014).

[34] A. Peña, A. Girschik, F. Libisch, S. Rotter, and A. A. Chabanov, Nat. Commun. 5, 3488 (2014).
[35] B. Gérardin, J. Laurent, A. Derode, C. Prada, and A. Aubry, Phys. Rev. Lett. 113, 173901 (2014).

[36] A. Lagendijk, R. Vreeker, and P. de Vries, Phys. Lett. A 136, 81 (1989).

[37] J. X. Zhu, D. J. Pine, and D. A. Weitz, Phys. Rev. A 44, 3948 (1991).

[38] D. Akbulut, Measurements of strong correlations in the transport of light through strongly scattering materials, Ph.D. thesis, University of Twente, 2013.

[39] X. Cheng, C. Tian, and A. Z. Genack, Phys. Rev. B 88, 094202 (2013).

[40] L. Zhao, C. Tian, Y. P. Bliokh, and V. Freilikher, Phys. Rev. B 92, 094203 (2015).

[41] A. F. Oskooi, D. Roundy, M. Ibanescu, P. Bermel, J. D. Joannopoulos, and S. G. Johnson, Comput. Phys. Commun. 181, 687 (2010).

[42] D. A. G. Bruggeman, Ann. Phys. (Leipzig) 416, 636 (1935).

[43] J. C. M. Garnett, Philos. Trans. R. Soc. London Ser. A 205, 237 (1906).

[44] T. Strudley, T. Zehender, C. Blejean, E. P. A. M. Bakkers, and O. L. Muskens, Nat. Photon. 7, 413 (2013).

[45] D. E. Aspnes and A. A. Studna, Phys. Rev. B 27, 985 (1983).

[46] E. N. Leith and J. Upatnieks, J. Opt. Soc. Am. 52, 1123 (1962).

[47] M. Takeda, H. Ina, and S. Kobayashi, J. Opt. Soc. Am. 72, 156 (1982).

[48] S. Olver and R. R. Nadakuditi, arXiv:1203.1958v2.

[49] A. Goetschy and A. D. Stone, Phys. Rev. Lett. 111, 063901 (2013)

[50] V. A. Marćénko and L. A. Pastur, Math. USSR Sb. 1, 457 (1967).

[51] S. Sirca and M. Horvat, Computational Methods for Physicists (Springer, Berlin, 2012).

[52] J. Gómez Rivas, R. Sprik, C. M. Soukoulis, K. Busch, and A. Lagendijk, Europhys. Lett. 48, 22 (1999).

[53] E. Akkermans, P. E. Wolf, and R. Maynard, Phys. Rev. Lett. 56, 1471 (1986).

[54] D. Gloge, Appl. Opt. 10, 2252 (1971).

[55] David Yuk Kei Ko and J. C. Inkson, Phys. Rev. B 38, 9945 (1988)

[56] W. Press, S. Teukolsky, W. Vetterling, and B. Flannery, Numerical Recipes: The Art of Scientific Computing, 3rd ed. (Cambridge University Press, Cambridge, 2007).

[57] P. García, R. Sapienza, A. Blanco, and C. López, Adv. Mater. 19, 2597 (2007). 